\title{
Determinantes sociales y alimentarios asociados al crecimiento lineal del niño juarense en edad preescolar*
}

\section{Social Determinants and Food Associated with Linear Growth of Preschool Juarense's Child}

\section{Abrabam Wall Medrano ${ }^{1}$, Wilebaldo L. Martínez \\ Toyes $^{2}$ y Valente David Aguirre Flores ${ }^{3}$}

1 Nacionalidad: Mexicana. Grado: Doctorado en Ciencias. Especialización: Nutrición y Salud Comunitaria. Adscripción: Profesor investigador de la Universidad Autónoma de Ciudad Juárez adscrito al Instituto de Ciencias Biomédicas. Correo electrónico: awall@ uacj.mx

2 Nacionalidad: Mexicana. Grado: Candidato a Doctor en Estudios de Población. Especialización: Migración interna e internacional, Dinámica demográfica, Seguridad Pública y Vivienda. Adscripción: Profesor investigador de tiempo completo de la Universidad Autónoma de Ciudad Juárez adscrito al Instituto de Ciencias Sociales y Administración. Correo electrónico: wmartine@uacj.mx

3 Nacionalidad: Mexicana. Grado: Licenciado en Nutrición. Especialización: Nutrición. Adscripción: Tesista de la Maestría en Salud Pública de la Universidad Autónoma de Ciudad Juárez. Correo electrónico: poseidon_vd@yahoo.com.mx.

* Resultados de investigación del proyecto financiado parcialmente por UACJ-FOMIX CONACYT-Gobierno del Estado de Chihuahua (CHIH-2008-C01-92093).

Fecha de recepción: 15 de junio de 2011

Fecha de aceptación: 17 de mayo de 2012 
Resumen

En la región norte, se cree que hay más población con talla alta. Sin embargo, la continua mezcla genética y el fenómeno migratorio parece haber afectado la prevalencia de talla alta con baja. Objetivo. Estudiar los factores socioeconómicos y alimentarios asociados de manera diferencial a la talla alta (TA) en preescolares de Ciudad Juárez en comparación a la talla baja (TB). Métodos. Estudio transversal retrospectivo en 295 niños $<5$ años de Ciudad Juárez. La estimación del peso $(\mathrm{P})$ y talla $(\mathrm{T})$ y puntajes $\mathrm{Z}$ $\mathrm{P} / \mathrm{E}, \mathrm{P} / \mathrm{T}$ y $\mathrm{T} / \mathrm{E}$ de cada niño y los factores socio demográficos y alimentarios familiares, se realizaron con técnicas y cuestionarios estandarizados y validados. Las diferencias en determinantes sociales fueron estudiados en niños altos $(Z-T / E>2 D E)$ y bajos (Z-T/E $>2 \mathrm{DE})$. Resultados. La prevalencia de TA y TB fue de 4.1 y 11.2, superiores a la media estatal. En comparación a la talla normal, la talla alta se relacionó a una complexión delgada (emaciación), mayor alimentación alergénica en el primer año de vida, tener dos padres originarios de Chihuahua $(\chi 2>4.0, \mathrm{p}<0.05)$. Percibir menos de 5 salarios mínimos en el hogar, pero menor probabilidad de inseguridad alimentaria, también se asoció con la talla alta. Con excepción de la proporción de padres de chihuahua y el haber recibido alimentación alergénica en el primer año, todas las demás variables (más desviaciones nutricionales negativas e inseguridad alimentaria familiar, pero menor percepción económica) justificaron la presencia de talla baja. Tanto la talla baja como la alta se asociaron a menor proporción de niños alimentados exclusivamente al seno materno. Conclusiones. Los factores socioeconómicos y alimentarios aquí estudiados parecen tener influencia sobre la talla de los niños, mismas que pueden ser directamente modificables por los padres o por el sistema económico.

Palabras claves: Preescolar, estado nutricio, alimentación, talla alta.

Abstract

In the northern region, it is believed that there are more people with high size. However, continuous genetic mixing and the migration phenomenon seems to have affected the prevalence of high stature with low. Objective. Study food and socio-economic factors associated differential way to the high stature (TA) preschool children in Ciudad Juárez in comparison to low size (TB). Methods. Retrospective cross-sectional study in 295 children < 5 years of Ciudad Juárez. Results. The prevalence of TA and TB was 4.1 and 11.2, higher than the State average. With the exception of the proportion of parents of Chihuahua and have received food allergen in the first year, all the other variables (more negative nutritional deviations and household food insecurity but minor economic perception) justified the presence of low stature. Both the high and low size were associated with lower proportion of children fed exclusively to the womb. Conclusions. Socioeconomic factors and food studied seem to have influence on the stature of children, same that can be directly modifiable by the parents or the economic system.

Key words: Preschool, claims status, diet, size high. 


\section{Introducción}

Según la Encuesta Nacional de Salud y Nutrición (ENSANUT) del 2006, 4.7, 1.2 y 0.2 millones de niños mexicanos $<5$ años se clasificaron con bajo peso (peso/edad $<-2 Z$ ), talla baja o desmedro (talla/ edad $<-2 Z$ ) y emaciación (peso/talla $<-2 Z$ ), respectivamente. Lo anterior significó importantes reducciones con respecto a lo reportado en la Encuesta Nacional de Nutrición del 1999, en todos estos indicadores a nivel nacional, pero para el caso norte no necesariamente. Los niños con desnutrición aguda en los estados como Chihuahua, van en franco descenso, aunque la mortalidad relacionada a ello, continúa siendo un problema de salud pública. Para el caso Juarense, los datos sobre la prevalencia de las distintas formas de desnutrición en el niño en edad preescolar son todavía muy escasos (Cárdenas, 2009). El análisis cauteloso de algunos estudios realizados por el cuerpo académico en salud comunitaria de la Universidad Autónoma de Ciudad Juárez, permite señalar que la prevalencia de bajo peso, talla baja y emaciación podría andar entre un siete a once por ciento. Por esto, se hace necesario un estudio más exhaustivo de estos indicadores en poblaciones infantiles que asisten a guarderías, jardines de niños y a otros servicios de cuidado diario de tipo ambulatorio.

A nivel nacional, las principales desviaciones en talla ocurren antes de que el niño cumpla los dos años, teniendo como resultado un $17.8 \%$ de prevalencia de talla baja en niños en edad preescolar (ENSANUT 2006) y manteniéndose esta casi hasta la adolescencia. La presencia de niños con talla baja perteneces a las familias más pobres (casi $48 \%$ en el status socioeconómico más bajo y de solo $4.6 \%$ en el status más alto). El Censo Nacional de Talla (CNT, 2004) reveló que los niños Chihuahuenses con talla baja representaban un $4.2 \%$ y $2.9 \%$ en niños de primer y sexto grado de primaria (Nacional 8.9 y 8.13 respectivamente). La talla baja en Chihuahua se acentúa particularmente en municipios rurales y zonas conurbanas y no precisamente en las ciudades grandes (ejemplo Juárez y Chihuahua) donde se ubican más del $50 \%$ de estos niños (Figura 1). Sin embargo y como se señaló anteriormente, los estudios sobre crecimiento lineal en niños Chihuahuenses 
en edad preescolar no solo son escasos, sino que arrojan cifras muy distintas, que no permiten evidenciar claramente la magnitud de este problema.

Figura 1 Cartografía de la talla baja a nivel municipal en Chihuahua 1994-2004.

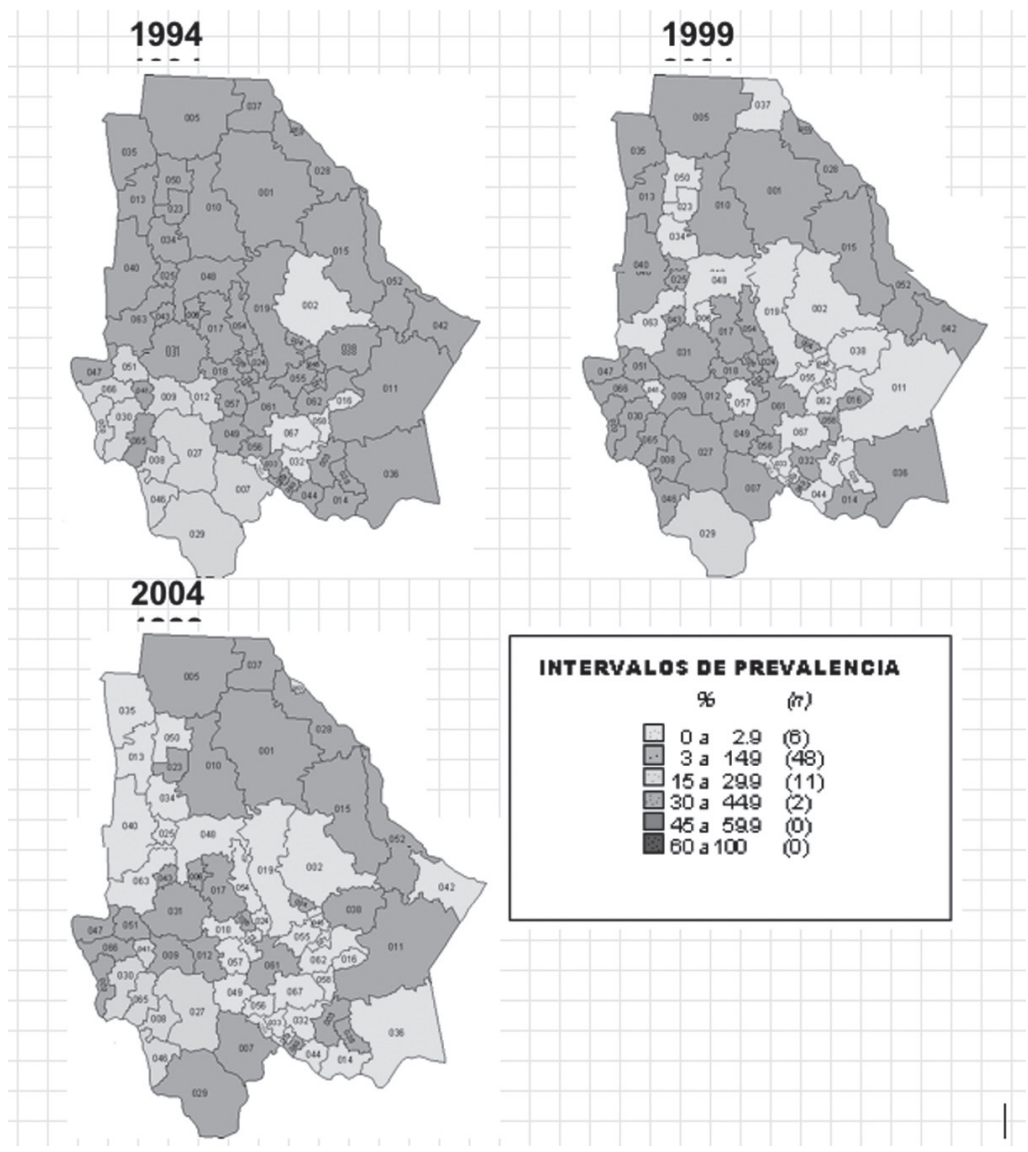

Fuente: CNT, 2004

Por otra parte, la talla alta se define como la talla para la edad situada dos desviaciones Standard (T/E $>2 Z$ ) por encima de la media para una población de referencia internacional. Las variantes normales de 
talla alta son el caso más frecuente (Leung y Robson, 1995). En condiciones normales, la talla alta esta determinada genéticamente (tallas familiares) con una característica velocidad de crecimiento incrementada en los 2 a 3 primeros años (Rial et al., 2001). Esto se presenta en familias con antecedentes familiares de pubertad temprana y talla adulta normales, y se caracterizan por una maduración ósea avanzada para la edad, pero concordante con la talla, sin alteraciones en los valores séricos de hormona de crecimiento, ni esteroides sexuales.

En sí misma, la talla alta familiar y el crecimiento acelerado no se definen como enfermedades. Por lo tanto, no han recibido mayor atención desde el punto de vista nutricional y epidemiológico. Sin embargo, la continua mezcla genética que se produce en México por el fenómeno migratorio y algunos otros determinantes sociodemográficos, ha ocasionado que la prevalencia de talla alta en los estados del norte, en donde en el pasado se suponía alta, se reduce cada vez más. En Chihuahua, por ejemplo, por cada niño $<5$ años con talla alta, hay 4 con talla baja, siendo en promedio para los estados del norte y a nivel nacional esta relación de 1 por cada 2 y 6 , respectivamente (Figura 2). 
Figura 2. Distribución de talla alta y baja en menores de 5 años a nivel nacional

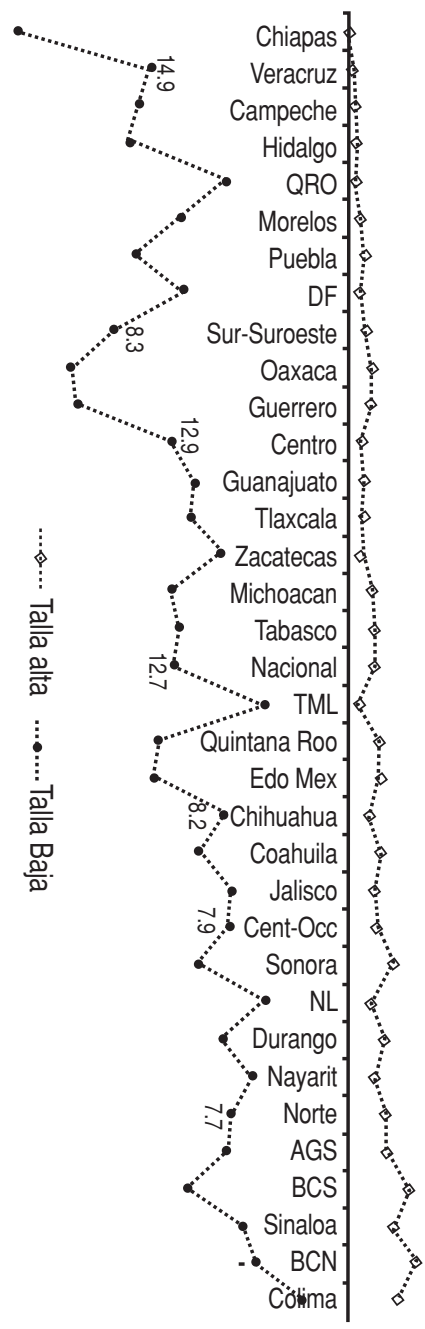

Fuente: ENSANUT, 2006 
Resulta importante señalar en este punto que en la estimación de prevalencias poblacionales, se emplean muestras probabilísticas que implican una mezcla de niños con muchos y muy diversos orígenes por lo que, en la prevalencia actual para el estado de Chihuahua se pueden estar incluyendo hijos de poblaciones migrantes. Ejemplo de esto son los niños de origen veracruzano, el principal grupo migrante del sur en la entidad, la relación talla alta vs. baja es de 1:37 mientras que para el caso Chihuahuense lo es de a 1:1.4 (Figura 2). Sin embargo, en un estudio previo (Wall-Medrano et al., 2008) se reporto que el origen familiar es un determinante de desnutrición en preescolares de Ciudad Juárez, pero la mejora en la economía familiar corrige dicho fenómeno. En este estudio se evalúa la relación de diversos factores socioeconómicos y alimentarios posiblemente asociadas a la desviación positiva y negativa de la talla de preescolares juarense.

\section{Materiales y métodos}

La pregunta de investigación que definió la propuesta metodológica fue: ¿Cuáles son los factores socioeconómicos y alimentarios que inciden en el desarrollo estatural de los preescolares juarenses?. La pertinencia del estudio se baso en el hecho de que existen todavía muy pocas investigaciones que relacionen el estado nutricio y muy particularmente en la presencia de desnutrición crónica (baja talla) para la población preescolar juarense. Más aun, estudios que relacionen este fenómeno con sus determinantes sociales, son todavía más escasos sobre en todo en época de crisis económica. Así, para lograr el objetivo planteado, a continuación se describe la muestra y metodología empleada en este trabajo.

\section{Muestra y Criterios de Inclusión.}

Se realizó un estudio transversal retrospectivo en población preescolar perteneciente a jardines de niños públicos y privados, además de las guarderías de los centros comunitarios participantes. Al selec- 
cionar dichas instituciones no se consideró la distribución geográfica ni el nivel socioeconómico, con el fin de evitar desviaciones de la muestra. La selección fue al azar, permitiendo recabar una muestra de 295 niños del total de niños inscritos en guarderías y jardines municipales. Los criterios de inclusión fueron niños menores de 5 años de edad pertenecientes a jardines de niños y guarderías de Ciudad Juárez en buen estado de salud general. Se consideró la aprobación oportuna por parte de las autoridades escolares correspondientes y padres de familia, mediante un consentimiento escrito para la obtención de información y toma de medidas antropométricas. Todos los procedimientos analíticos y de campo cumplieron con los requisitos estipulados en la declaración de Helsinki.

\section{Evaluación antropométrica.}

Siguiendo técnicas estandarizadas se obtuvieron las mediciones del peso corporal con básculas digitales (Tanita mod. 682) y la talla con un estadímetro portátil (Seca mod. 208). Una vez hechas las mediciones, la clasificación del estado nutricional de los participantes se realizó en base a los indicadores antropométricos talla para la edad (T/E), peso para la talla $(\mathrm{P} / \mathrm{T})$, peso para la edad $(\mathrm{P} / \mathrm{E})$ e índice de masa corporal [peso (kg)/Talla2 (m2)] para la edad (IMC/E), según la Organización Mundial de la Salud utilizando el programa EPIINFO (6.0), basado en las curvas de referencia y crecimiento estándar de niños americanos desarrollados por el Centro Nacional para Estadísticas en Salud (NCHS, por sus siglas en ingles) de los Estados Unidos de América y adoptadas por la Organización Mundial de La Salud (OMS) como patrón internacional (Kuczmarski et al., 2000) y evaluados dentro del programa Epi Info 6.04d ( http://www.cdc.gov/epiinfo). Los indicadores T/E, P/E. P/T e IMC/E ubicados entre $+2 Z$ y $-2 Z$ (desviaciones estándar) fueron considerados como normales. El peso bajo, talla baja y la emaciación fueron definidas por los indicadores $\mathrm{P} / \mathrm{E}, \mathrm{T} / \mathrm{E}$ y $\mathrm{P} / \mathrm{T}$ por $<-2 \mathrm{Z}$, mientras que el sobrepeso-obesidad $(\mathrm{Sp} / \mathrm{Ob})$ fue defi- 
nido por los indicadores $\mathrm{P} / \mathrm{T}$ (en menores de 2 años) e IMC/E (en los mayores de 2 años) $>2 Z$ y la talla alta se definió a T/E $>2 Z$.

\section{Características socioeconómicas familiares y alimentación en el} primer año de vida.

Para estudiar la relación entre el estado nutricional actual del niño con las características socioeconómicas familiares y prácticas de lactancia y ablactación en el primer año de vida, se utilizaron las mismas preguntas realizadas en la ENSANUT 2006 Se utilizó este cuestionario con fines comparativos una vez que se publicaron los hallazgos encontrados a nivel nacional. El cuestionario estuvo estructurado para ser aplicado por entrevista a profundidad con el tutor del niño.

\section{Análisis Estadístico.}

Se utilizaron medidas descriptivas de tendencia central para la exploración inicial de los datos con distribución normal (CI 95\%). El análisis de las diferencias estadísticas para las distintas variables continuas (F Fisher) o no paramétricas (Chi cuadrada) analizadas, fue realizado por comparación entre individuos con talla normal (grupo 1) vs. Aquellos que tenían talla baja (grupo 2) o alta (grupo 3), a un nivel de significancia del 95\% $(\mathrm{p}<0.05)$.

\section{Resultados}

Características sociodemográficas, culturales y económicas.

Los datos más importantes tanto de los 295 participantes como las de su familia se mencionan a continuación. El 25\% de las madres cursaron menos de 10 años de escolaridad y el 37\% haber llegado a licenciatura. Solo el $14 \%$ del número de ambos padres no trabaja actualmente y cerca de 30\% tenían más de un empleo. Más de la mitad de los hogares percibían mensualmente mas de $\$ 5,000$ pesos mensuales, destinando el $50 \%$ de este a alimentación. En cuanto a la inmigración, 
la población que dijo ser originaria de Ciudad Juárez era tres veces más grande que la que refirió venir de otro lugar. Alrededor de la tercera parte de la muestra, vivían en cuartos de vecindad, departamentos o en casa prestada. En su mayoría estas casas tenían techo de material o losa de concreto, el piso de mosaico y paredes hechas con tabique o ladrillo. Todas las casas contaban con los servicios básicos para su función, como lo es la energía eléctrica, drenaje y baño exclusivo del hogar, pero solo 1/5 contaba con más de dos televisores. Estas características permiten concluir que los hogares eran en su mayoría de nivel socioeconómico medio-bajo.

\section{Evaluación del estado nutricio de los participantes del estudio.}

La comparación con las prevalencias estatal y nacional de las distintas desviaciones del estado nutricio infantil en la muestra aquí estudiada $(n=295)$ se muestran en la Figura 3. Con excepción de la talla baja a nivel estatal, la prevalencia de todas las desviaciones nutricionales negativas (bajo peso, emaciación, sobrepeso/obesidad y talla baja) y de talla alta fue superior en este estudio. Específicamente, la prevalencia de TA y TB fue de 4.1 y 11.2, superiores a la media estatal (8.2 y $2.0 \%$, respectivamente).

Figura 3. Estado nutricional de niños $<5$ años. Bajo Peso (BP), emaciación (EM), sobrepeso/obesidad ( $\mathrm{Sp} / \mathrm{Ob})$, talla baja (TB), talla alta (TA).

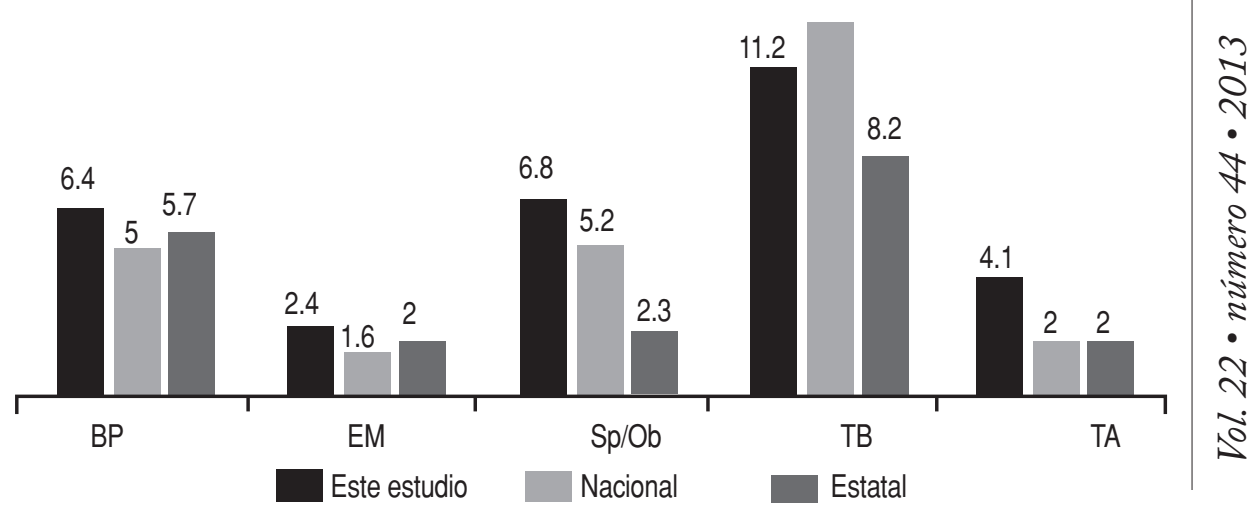


Factores asociados a las desviaciones en talla.

Por otra parte, las características socioeconómicas y alimentarias asociadas a la talla alta $(\mathrm{T} / \mathrm{E}>2 Z)$ y baja $(\mathrm{T} / \mathrm{E}<2 \mathrm{Z})$ y normal, se muestran en la Tabla 1.

Tabla 1. Características socioeconómicas y alimentarias asociadas a la Talla de preescolares juarenses (\%).

\begin{tabular}{|c|c|c|c|}
\hline \multirow[b]{2}{*}{ Característica } & \multicolumn{3}{|l|}{ Talla } \\
\hline & $\begin{array}{l}\text { Normal } \\
(n=250)\end{array}$ & $\begin{array}{c}\text { Alta } \\
(n=12)\end{array}$ & $\begin{array}{c}\text { Baja } \\
(n=33)\end{array}$ \\
\hline Bajo Peso & $3.6 \mathrm{a}$ & $0.0 \mathrm{a}$ & $30.3 \mathrm{~b}$ \\
\hline Emaciación & $2.4 \mathrm{a}$ & $8.3 \mathrm{~b}$ & $0.0 \mathrm{c}$ \\
\hline Sobrepeso/Obesidad & $5.6 \mathrm{a}$ & $8.3 \mathrm{a}$ & $15.2 \mathrm{~b}$ \\
\hline $\begin{array}{l}\text { Hogar con } \\
\text { inseguridad alimentaria }\end{array}$ & $11.6 \mathrm{a}$ & $8.3 \mathrm{a}$ & $21.2 \mathrm{~b}$ \\
\hline Alimentación alergénica & $17.6 \mathrm{a}$ & $25.0 \mathrm{~b}$ & $12.2 \mathrm{a}$ \\
\hline Menos de 5 salarios mínimos & $43.6 \mathrm{a}$ & $66.7 \mathrm{~b}$ & $60.6 \mathrm{~b}$ \\
\hline 1 padre de chihuahua & $33.2 \mathrm{a}$ & $25.0 \mathrm{a}$ & $33.3 \mathrm{a}$ \\
\hline 2 padres de chihuahua & $34.0 \mathrm{a}$ & $50.0 \mathrm{~b}$ & $33.3 \mathrm{a}$ \\
\hline $\begin{array}{l}\text { Lactancia materna ex- } \\
\text { clusiva (LME) }\end{array}$ & $18.8 \mathrm{a}$ & $0.0 \mathrm{~b}$ & $3.1 \mathrm{c}$ \\
\hline
\end{tabular}

Diferente superíndice indica diferencias significativas entre las deviaciones en talla vs. La talla normal $(\chi 2>4.0, p<0.05)$

En comparación a la talla normal, la talla alta se asocio a mayor prevalencia de emaciación, mayor alimentación alergenica en el primer año de vida, tener dos padres originarios de Chihuahua $(\chi 2>4.0$, $\mathrm{p}<0.05)$. Percibir menos de 5 salarios mínimos en el hogar, pero menor probabilidad de inseguridad alimentaria, también se relacionó con la talla alta. Con excepción de la proporción de padres de chihuahua y el haber recibido alimentación alergenica en el primer año, todas las demás variables (más desviaciones nutricionales negativas e inseguridad alimentaria familiar, pero menor percepción económica) se asociaron 
a la talla baja. Tanto la talla baja como la alta se asociaron a menor proporción de niños alimentados exclusivamente al seno materno.

\section{Discusión}

El propósito de esta investigación fue el estudiar los factores socioeconómicos y alimentarios relacionados con la talla alta (TA) y baja (TB) en los niños preescolares de Ciudad Juárez. Según la Encuesta Nacional de Talla (CNT, 2004) en Chihuahua la TB en niños de primer año de primaria se distribuye principalmente entre poblaciones rurales y zonas conurbanas. En la ENSANUT 2006 se indicó que en este estado hay un preescolar con TA por cada 4 con TB. Aquí se reporta que esta relación fue de 1 por cada 3 niños, pero la prevalencia individual de TA y TG se encuentra muy por encima de la media estatal. Por si esto fuera poco, la prevalencia de sobrepeso/ obesidad en la población analizada (6.8\%) es superior a las medias estatal (5.2\%) y nacional (2.3\%). De lo anterior se desprende que los problemas de desnutrición crónica (TB) concurrente a la presencia de sobrepeso y obesidad en esta población preescolar, coincide con lo reportado por Fernald y Neufeld $(2007,623-32)$ para el preescolar de zona rural.

Organismos internacionales señalan que lo más importante para reducir la prevalencia de TB es mejorar las condiciones socioeconómicas, ambientales y nutricionales en las que se desenvuelve el niño (Tanumihardio et al., 2007, 1966-72). El ingreso familiar constituye un factor fundamental para el desarrollo de los niños. En tal sentido, un mayor ingreso puede facilitar el acceso de la familia hacia medios que permitan mantener un nivel nutricional adecuado de los niños. Por otro lado, una alimentación adecuada dentro del hogar tiene un efecto positivo sobre las condiciones de salud del niño, sobre su habilidad para aprender, comunicarse, pensar analíticamente, socializar efectivamente y adaptarse a nuevos ambientes y personas. Es esencial un equilibrado aporte nutricional para obtener un adecuado estado de salud, un óptimo crecimiento y desarrollo físico y psicosocial, y 
además ayudar al establecimiento de hábitos alimentarios saludables que permitan prevenir problemas de salud en la edad adulta.

En términos muy generales, la relación entre la pobreza y desnutrición crónica tienen una justificación teórica aparentemente sencilla. Los ingresos insuficientes provocan la limitación en la compra de alimentos saludables contenidos en la canasta básica y otros satisfactores. Esta situación produce deficiencias nutricionales en la población de bajo nivel socioeconómico, por lo que resulta común encontrar inseguridad alimentaria familiar (Tanumihardio et al., 2007: 1966-72; Díaz et al, 2002: 284-289) y con ello efectos en el crecimiento físico y composición corporal del niño en edad preescolar (Acuña y Solano, 2009:5-11). Con relación al estrato social, la literatura científica formal apunta a que los niños de estratos socioeconómicos bajos son generalmente más pequeños y maduran más lentamente que los niños de estratos altos. En este estudio se observo una relación de más de 20 puntos porcentuales en la proporción de hogares con percepción menor de 5 salarios mínimos, entre los niños con talla normal y aquellos con baja o alta. Sin embargo, la presencia de inseguridad alimentaria familiar en los hogares de aquellos con talla baja, fue un sello distintivo frente a los otros grupos.

Así, los aparentes efectos positivos representados por un mejor ingreso económico y la consecuente disminución de inseguridad alimentaria familiar que presentaron pueden mejorar la probabilidad de crecimiento lineal de los niños juarenses. Estos resultados concuerdan con los de Kain et al. (2003: 1013-20) que asocian la prevalencia de talla alta de niños de 6 años con el nivel socioeconómico y demográfico con que cuentan las familias. Esto podría estar indicando que, la inseguridad alimentaria que experimentan los hogares, cuya percepción se encuentra por debajo de los 5 salarios mínimos obedece posiblemente a una inadecuada política de precios aplicable a la canasta básica en esta región, trayendo como consecuencia el intercambio de alimentos más baratos, pero de menor valor nutricional, cuyo impacto se ve reflejado en el crecimiento estatural de estos niños. Sin embargo, esta hipótesis merece una exploración posterior en futuras investigaciones. 
La mayor expresión del crecimiento esta determinada por factores ambientales, entre los que destacan los usos y costumbres alimentarias como el patrón de lactancia y alimentación complementaria en el primer año de vida. Durante el primer año, el niño adquiere los nutrimentos para la síntesis de nuevos tejidos y mantenimiento de su sistema inmunitario lo que permite que el crecimiento sea apropiado si se satisfacen los requerimientos de los nutrimentos necesarios (de Onis et al., 2009: 47-53). Al respecto, existen elementos críticos en el crecimiento de los niños: el tipo de lactancia que se establece, y la duración de la lactancia, la edad de inicio de alimentación complementaria o ablactación y los alimentos utilizados en esta ultima (Owen et al, 2005: 1298-1307). En este estudio se encontró que la lactancia materna exclusiva se relaciono mas a tener talla normal que TA o TB mientras que la introducción de alimentos potencialmente alergénicos como el huevo, pescados y mariscos, a tener TA. Previendo que en la promoción de la lactancia materna exclusiva (LME) y la adecuada introducción de alimentos complementarios en el primer año de vida resulta indispensable considerar los factores culturales y socio-demográficos de la madre (Guerrero et al., 1999: 323-30), resulta indispensable estudiar mas a fondo esta problemática en la mujer de esta frontera previo a su inicio.

Por último, el análisis de los factores asociados a la talla alta reveló que el componente genético es importante. Es de creencia popular que los individuos del norte del país tienen una mayor talla debido a que existe un mayor ingreso económico, por lo tanto, destinan la mayor parte de sus ingresos a la alimentación. Sin embargo, En este estudio se demostró que la prevalencia de talla alta se relaciona con los nacimientos de parejas cuyo origen es Chihuahuense. En un estudio previo al que aquí se reporta se encontró que el origen familiar es un determinante de desnutrición en preescolares de Ciudad Juárez, pero que la mejora en la economía familiar corrige dicho fenómeno (WallMedrano et al., 2008: 1-13). Ambos estudios refuerzan la necesidad de investigar desviaciones positivas y negativas de una variable biológica (en este caso la estatura) rompiendo con el esquema tradicional de enfoque de los estudios nutricionales, siempre hacia el déficit. Este 
tipo de investigaciones permite completar los estudios realizados a la talla baja y postular a la luz de la evidencia científica, los determinantes sociales asociados de manera diferencial a la TA y TB para promover un adecuado crecimiento y mejorar la calidad de vida del preescolar de nuestra región.

En resumen, existen variables que pueden influenciar el status nutricional de los niños y que pueden ser directamente controladas por los padres o del sistema económico. Recibir una alimentación adecuada en los primeros años de vida, vivir en un hogar seguro que cuente con todos los servicios básicos y tener un mejor ingreso económico, son factores fundamentales para el desarrollo físico, social y emocional de los niños. La infraestructura del hogar y el combate a la pobreza alimentaria, son factores que tiene un efecto positivo significativo sobre el estado de salud de los niños, y en la medida en que esta sea accesible para todos y se encuentre en un estado óptimo, contribuirá a mejorar su desarrollo.

\section{Referencias}

Acuña, G.I., Solano, R.L. (2009). "Situación socioeconómica, diagnóstico nutricional antropométrico y dietario en niños y adolescentes de Valencia, Venezuela”. An Venez Nutr, num. 1, Vol. 22, pp. 5-11.

Cárdenas, B.K.A. (2009). Factores socioeconómicos y alimentarios asociados al niño alto juarense en edad preescolar. Tesis Profesional. México: Universidad Autónoma de Ciudad Juárez-Instituto de Ciencias Biomédicas, pp. 1-31.

CNT. IV Censo Nacional de Talla en escolares de primero y sexto grado. México: SSA/ SEP/ DIF/ INCMNSZ/ INEGI, 2005.

Díaz N., Páez, M.C., Solano, R.L. (2002). "Situación nutricional por estrato social en niños escolarizados venezolanos". Acta Científica Venezolana, Vol. 53, pp. 284-289.

De Onis, M.; Garza, C.; Onvango, A.W.; Rolland-Cachera, M.F.; le Comité de nutrition de la Société Franchise de pédiatrie. (2009). "Les standards de croissance de l'Organisation mondiale de la 
santé pour les nourrissons et les jeunes enfants". Arch Pediatr, num. 1, Vol. 16, pp. 47-53.

Sepúlveda, A.J.; Rivera, D.J.; Shamah, L.T. (1999). Encuesta nacional de nutrición 1999. Estado nutricio de mujeres y niños. México: Instituto Nacional de Salud Pública (INSP), pp. 1-315, ISBN: 968-6502-54-8.

Oláis-Fernández, G.; Rivera-Dommarco, J.; Shamah-Levy, T.; Rojas, R.; Villalpando-Hernández, S.; Hernández-Ávila, M.; et al. Encuesta nacional de salud y nutrición 2006. (2006). México: México: Instituto Nacional de Salud Pública (INSP), pp. 1-132, ISBN: 970-9874-20-9.

Fernald, L.C.; Neufeld, L.M. (2007). "Overweight with concurrent stunting in very young children from rural Mexico: prevalence and associated factors". Eur J Clin Nutr, Vol. 61, pp. 623-632.

Guerrero, M.L., Morrow, R.C., Calva, J.J., Ortega-Gallegos, H., Weller, S.C., Ruiz-Palacios, G.M., Morrow, A.L. (1999). "Rapid ethnographic assessment of breastfeeding practices in periurban Mexico City". Bull World Health Organ, num. 4, Vol. 77, pp. 323-330.

Kain, J.; Uauy, R.; Lera, L.; Taibo, M.; Espejo, F.; Albala, C. (2003). "Evolución del estado nutricional de escolares chilenos de 6 años". Rev Med Chile, Vol. 133, pp. 1013-1020.

Kuczmarski R.L., Ogden C.L, Grummer-Strawn L.M., Flegal K.M., Guo S.S.,Wei R. et al. (2000). CDC Growth charts United States. Advanced data from vital and health statistic. Hyattsville, Maryland: National Center for Health Statistic (NCHS), num. 314, pp. $1-27$.

Leung, A.K.C., Robson, W.L.M. (1995). "Evaluating tall children". Can Fam Physician, Vol. 41, pp. 457-468.

Rial, J., Rodríguez I., González J., López R. (2001). “Diagnóstico Diferencial del Hipercrecimiento". BSCP Can Ped, num. 1, vol. 26, pp. 1-9.

Tanumihardio, S.A.; Anderson, C.; Kaufer-Horwitz, M.; Bode, L.; Emenaker, N.J; Hagg, A.M.; Satia, J.A.; Silver, H.J.; Stadler, D.D. (2007). "Poverty, Obesity, and Malnutrition: An International 
Perspective Recognizing the Paradox". J Am Diet Assoc, num. 11, vol. 107, pp. 1966-1972.

Wall-Medrano, A.; Arreola-González, R.; Rodríguez-Tadeo, A. (2008). "Relación entre el origen y economías familiares con el estado nutricio de niños en edad preescolar de Ciudad Juárez, Chihuahua". Avances, Vol. 181. 